\title{
Provision of rapid and specific ex vivo diagnosis of central nervous system lymphoma from rodent xenograft biopsies by a fluorescent aptamer
}

\author{
Joseph Georges, DO, PhD, ${ }^{7-9}$ Xiaodong Qi, PhD,, 45 Xiaowei Liu, PhD, ${ }^{4}$ Yu Zhou, MS, ${ }^{4,5}$ \\ Eric C. Woolf, PhD, ${ }^{10}$ Amber Valeri, D0,7,8 Zein Al-Atrache, DO, PhD, ${ }^{7}$ Evgenii Belykh, MD, PhD,, 10 \\ Burt G. Feuerstein, MD, PhD, ${ }^{2,3}$ Mark Preul, MD, ${ }^{9,10}$ Adrienne C. Scheck, PhD, ${ }^{3}$ Mark Reiser, PhD, ${ }^{6}$ \\ Trent Anderson, PhD, ${ }^{2}$ Jonas Gopez, MD, ${ }^{12}$ Denah Appelt, PhD, ${ }^{7}$ Steven Yocom, DO, 7,8 \\ Jennifer Eschbacher, MD, ${ }^{11}$ Hao Yan, PhD, ${ }^{4}$ and Peter Nakaji, MD ${ }^{1}$
}

Departments of ${ }^{1}$ Neurosurgery, ${ }^{2}$ Neurology, and ${ }^{3} \mathrm{Child}$ Health, University of Arizona, College of Medicine, Phoenix, Arizona; ${ }^{4}$ The Biodesign Institute, ${ }^{5}$ School of Molecular Sciences, ${ }^{6}$ School of Mathematics and Statistical Sciences, Arizona State University, Tempe, Arizona; ${ }^{7}$ Department of Neurosurgery, Philadelphia College of Osteopathic Medicine, Philadelphia, Pennsylvania; ${ }^{8}$ Department of Neurosurgery, Cooper University Health Care, Camden, New Jersey; ' ${ }^{2}$ epartment of Neurosurgery, ${ }^{10}$ NeuroOncology Research, ${ }^{11}$ Division of Neuropathology, Barrow Neurological Institute, Phoenix, Arizona; and ${ }^{12}$ Department of Neurosurgery, Abington Hospital-Jefferson Health, Abington, Pennsylvania

OBJECTIVE Differentiating central nervous system (CNS) lymphoma from other intracranial malignancies remains a clinical challenge in surgical neuro-oncology. Advances in clinical fluorescence imaging contrast agents and devices may mitigate this challenge. Aptamers are a class of nanomolecules engineered to bind cellular targets with antibodylike specificity in a fraction of the staining time. Here, the authors determine if immediate ex vivo fluorescence imaging with a lymphoma-specific aptamer can rapidly and specifically diagnose xenografted orthotopic human CNS lymphoma at the time of biopsy.

METHODS The authors synthesized a fluorescent CNS lymphoma-specific aptamer by conjugating a lymphomaspecific aptamer with Alexa Fluor 488 (TD05-488). They modified human U251 glioma cells and Ramos lymphoma cells with a lentivirus for constitutive expression of red fluorescent protein and implanted them intracranially into athymic nude mice. Three to 4 weeks postimplantation, acute slices (biopsies, $n=28$ ) from the xenografts were collected, placed in aptamer solution, and imaged with a Zeiss fluorescence microscope. Three aptamer staining concentrations $(0.3,1.0$, and $3.0 \mu \mathrm{M})$ and three staining times $(5,10$, and 20 minutes) followed by a 1-minute wash were tested. A file of randomly selected images was distributed to neurosurgeons and neuropathologists, and their ability to distinguish CNS lymphoma from negative controls was assessed.

RESULTS The three staining times and concentrations of TD05-488 were tested to determine the diagnostic accuracy of CNS lymphoma within a frozen section time frame. An 11-minute staining protocol with 1.0- $\mu \mathrm{M}$ TD05-488 was most efficient, labeling $77 \%$ of positive control lymphoma cells and less than $1 \%$ of negative control glioma cells $(p<0.001)$. This protocol permitted clinicians to positively identify all positive control lymphoma images without misdiagnosing negative control images from astrocytoma and normal brain.

CONCLUSIONS Ex vivo fluorescence imaging is an emerging technique for generating rapid histopathological diagnoses. Ex vivo imaging with a novel aptamer-based fluorescent nanomolecule could provide an intraoperative tumor-specific diagnosis of CNS lymphoma within 11 minutes of biopsy. Neurosurgeons and neuropathologists interpreted images generated with this molecular probe with high sensitivity and specificity. Clinical application of TD05-488 may permit specific intraoperative diagnosis of CNS lymphoma in a fraction of the time required for antibody staining.

https://thejns.org/doi/abs/10.3171/2020.4.JNS192476

KEYWORDS neurosurgery; fluorescence; aptamer; neuropathology; CNS lymphoma; frozen section; biopsy; molecular imaging; oncology

ABBREVIATIONS CNS = central nervous system; IHC = immunohistochemistry; RFP = red fluorescent protein; ROI = region of interest.

SUBMITTED September 13,2019. ACCEPTED April 23, 2020.

INCLUDE WHEN CITING Published online July 24, 2020; DOI: 10.3171/2020.4.JNS192476. 
$\mathrm{T}$ REATMENT for cancer patients often relies on definitive histopathological diagnosis from biopsies. ${ }^{1}$ However, diagnostic stains are often time-consuming and can delay patient-specific treatment plans, including decisions regarding resection. Brain tumors such as astrocytomas are often debulked in surgical candidates, whereas central nervous system (CNS) lymphoma is generally not surgically debulked and is best treated with chemoradiation. ${ }^{2}$ Preoperatively, CNS lymphoma can be indistinguishable from other malignant brain tumors with imaging modalities such as MRI and CT, and minimally invasive techniques such as flow cytometry can yield inconclusive results. ${ }^{3}$ Therefore, direct tissue sampling is often required to definitively diagnose CNS lymphoma.,

Open biopsy and stereotactic needle biopsy are common techniques for sampling brain tumors. ${ }^{6}$ Patients with tumors amenable to surgery typically undergo concomitant open biopsy with tumor resection. ${ }^{7,8}$ During open biopsy, a frozen section is often obtained early in the case to guide the surgical plan. If the frozen section suggests CNS lymphoma, the surgery is often halted. If the tumor diagnosis on frozen section is other than CNS lymphoma, the surgery proceeds to maximal safe resection. However, the delay from waiting for the frozen section result can unnecessarily extend surgery time. Furthermore, frozen sections occasionally fail to differentiate CNS lymphoma from tumors that benefit more from resection such as astrocytomas..$^{9,10}$ This denies information critical for good decision-making at open biopsy. These cases require special stains to obtain a specific diagnosis, a process that can take days to weeks. ${ }^{11}$ The lack of accurate and specific histopathological information can lead to inappropriate termination of surgery or resection of a tumor best treated without surgery. Therefore, rapid and specific intraoperative diagnosis of CNS lymphoma could improve surgical decision-making.

Immunohistochemistry (IHC) generates a definitive diagnosis of CNS lymphoma using antibodies against lymphoma-specific proteins, such as CD20. ${ }^{12}$ Unfortunately, IHC is a multistep staining procedure too slow for intraoperative feedback. Therefore, molecular probes that identify CNS lymphoma more efficiently than IHC may expedite diagnosis. ${ }^{3}$

Aptamers are a class of nanomolecules that bind molecular targets with antibody-like affinity. ${ }^{13,14}$ Unlike antibodies, aptamers are small and can readily diffuse through tissue samples to quickly bind their targets. Aptamers can be conjugated to fluorophores for molecular imaging and to chemotherapeutics for targeted therapy. ${ }^{3,15}$ Aptamers are possible reagents for histopathological tissue assessments; however, development of aptamer-based diagnostics remains in its infancy.

We previously described a lymphoma-specific, aptamer-based, conformational-switching molecular probe, TD05, that could identify human CNS lymphoma in animal brain tumor biopsies within 60 minutes. ${ }^{3}$ The binding location of this aptamer on B-cell lymphoma cells, its strong overlap with CD20 immunostaining, and its lack of binding to $\mathrm{T}$ cells have been previously reported by our group and others. ${ }^{3,16,17}$ Here, we optimize a protocol using this truncated aptamer to generate a tissue-specific diagnosis of CNS lymphoma in 20 minutes or less from the time of biopsy. We hypothesized that a fluorescent aptamer that identifies at least $80 \%$ of tumor cells with a falsepositive rate of less than $10 \%$ will provide histopathological identification of human CNS lymphoma from rodent xenograft brain tumor biopsies with IHC-like accuracy in a time frame similar to that for frozen sections.

\section{Methods}

\section{TD05-488 Preparation}

The DNA oligonucleotide, which had been isolated with high-performance liquid chromatography purification, was purchased from Integrated DNA Technologies Inc. A truncated version of the TD05 aptamer was used in our study. ${ }^{3}$ The sequence of the fluorophore-labeled aptamer is as follows: TD05-488: 5'/5Alex488N/AGGAGG ATAGTTCGGTGGCTGTTCAGGGTCTCCTCCT-3'.17,18 We diluted aptamer probes to 3,1 , and $0.3 \mu \mathrm{M}$ in aptamer binding buffer $\left(6 \mathrm{mM} \mathrm{MgCl}_{2}, 1.2 \mathrm{mM} \mathrm{CaCl}_{2}, 4.5 \mathrm{~g} / \mathrm{L}\right.$ glucose, and $0.2 \% \mathrm{NaN}_{3}$ in $1 \times$ phosphate-buffered saline buffer) and annealed by heating at $94^{\circ} \mathrm{C}$ for 5 minutes, followed by immediate chilling on ice for 10 minutes.

\section{Fluorescent Tumor Preparation}

Human glioma cells (U251) and human CNS lymphoma cells (Ramos) were acquired from American Type Culture Collection. We incubated U251 cells in DMEM supplemented with $10 \%$ fetal bovine serum (FBS) and Ramos cells in RPMI 1640 medium supplemented with $10 \% \mathrm{FBS}$ at $37^{\circ} \mathrm{C}$ in a humidified incubator with $5 \%$ carbon dioxide. We transduced U251 and Ramos cells with a lentivirus for stable expression of red fluorescent protein (RFP) under puromycin selection (Gentarget Inc.).

\section{Animals}

Nude mice $(n=8)$ were obtained from The Jackson Laboratory and housed at the Barrow Neurological Institute's animal care facilities. All experiments were performed under the guidelines and regulations set forth by the National Institutes of Health Guide for the Care and Use of Laboratory Animals and approved by the Institutional Animal Care and Use Committee of the Barrow Neurological Institute at St. Joseph's Hospital and Medical Center.

\section{Intracranial Implantation}

Nude mice (6-7 weeks of age) were anesthetized with an intraperitoneal injection of ketamine $(10 \mathrm{mg} / \mathrm{kg})$ and xylazine $(80 \mathrm{mg} / \mathrm{kg})$, placed in a stereotactic apparatus (Kopf Instruments), and incised over the cranial midline. A burr hole was made $0.1 \mathrm{~mm}$ posterior to the bregma and $2.3 \mathrm{~mm}$ to the right of the midline. A needle was inserted to a depth of $3 \mathrm{~mm}$ and withdrawn $0.4 \mathrm{~mm}$ to a depth of $2.6 \mathrm{~mm}$. Red fluorescent protein-expressing U251 or Ramos cells were infused over the course of 3 minutes. The burr hole was closed with bone wax, and the incision was sutured.

\section{Acute Brain Slices}

Rodents were deeply anesthetized with isoflurane $21-$ 

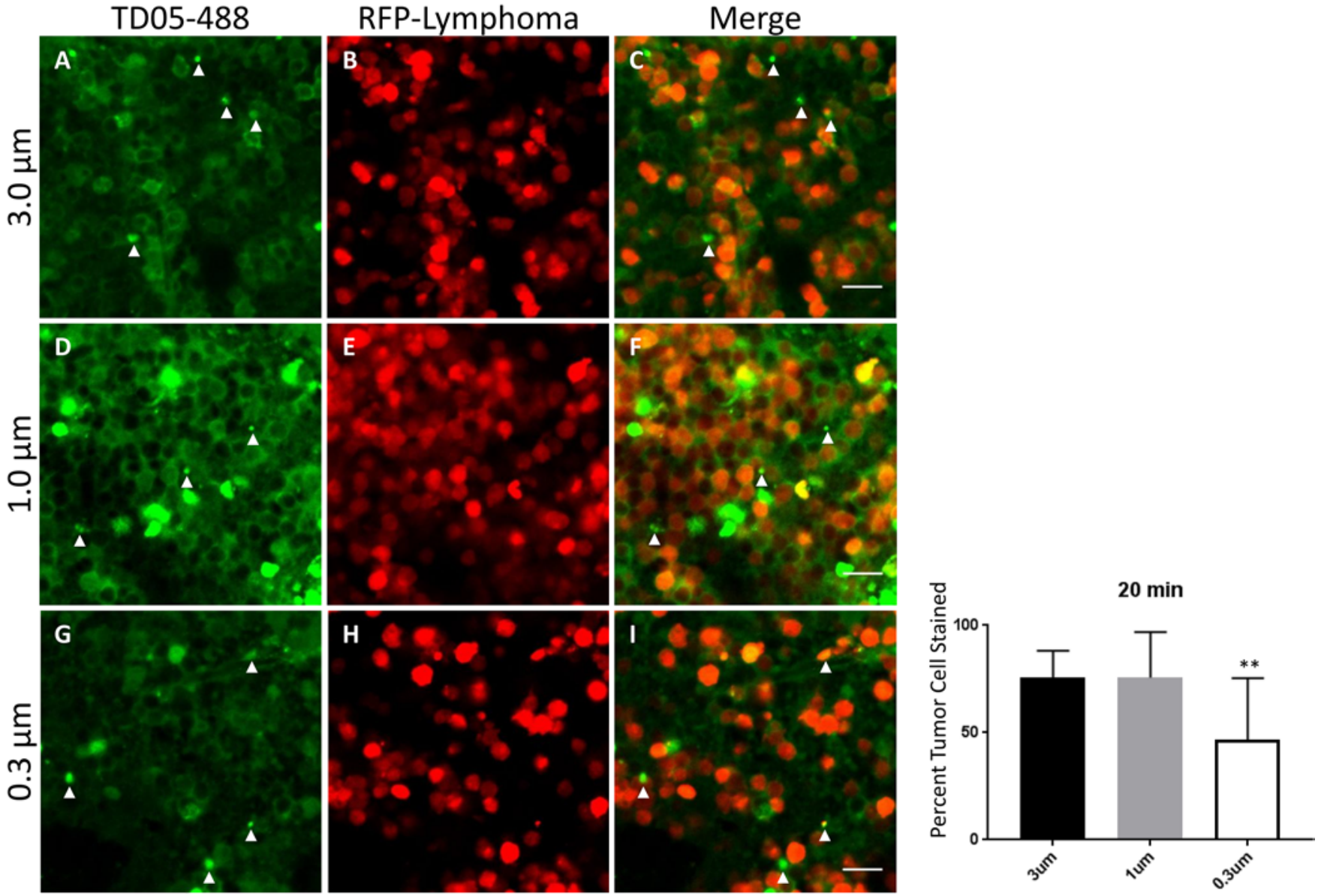

FIG. 1. Twenty-minute staining protocol. Images of xenograft biopsies with RFP-expressing tumor cells stained with 3.0- $\mu \mathrm{M}$ $(\mathbf{A}-\mathbf{C}), 1.0-\mu \mathrm{M}(\mathrm{D}-\mathbf{F})$, and $0.3-\mu \mathrm{M}(\mathbf{G}-\mathbf{I})$ TD05-488 aptamer. The 0.3- $\mu \mathrm{M}$ concentration identified significantly fewer tumor cells than the 1.0- and 3.0- $\mu \mathrm{M}$ concentrations. Fluorescent artifacts without ring-like staining were present across all aptamer samples (arrowheads). Bar $=20 \mu \mathrm{m} .{ }^{* *} p<0.001$.

28 days post-tumor implantation and rapidly decapitated. Their brains were immediately removed and sectioned into $350-\mu \mathrm{m}$ sections with a Leica VT1200 vibratome containing artificial cerebrospinal fluid (aCSF; in mM: $126 \mathrm{NaCl}, 26 \mathrm{NaHCO}_{3}, 2.5 \mathrm{KCl}, 1.25 \mathrm{NaH}_{2} \mathrm{PO}_{4}, 2 \mathrm{MgSO}_{4}$, $2 \mathrm{CaCl}_{2}$, and 10 glucose; $\left.\mathrm{pH} 7.4\right)$. Acute slices $(\mathrm{n}=28)$ were maintained at $37^{\circ} \mathrm{C}$ in aCSF until aptamer staining.

\section{Fluorescent Aptamer Staining Protocol}

The annealed aptamers were mixed with yeast transfer RNA (tRNA; $0.1 \mathrm{mg} / \mathrm{ml}$ ), which was used to block nonspecific binding. One milliliter of the prepared aptamer mixture was applied to submerse the acute tissue slices in a glass bottom dish and incubated on ice for 20, 10, or 5 minutes. Staining solution was then aspirated from the staining dish, and the tissue slices were quickly rinsed with $1 \mathrm{ml}$ of ice-cold aptamer binding buffer for 1 minute before fluorescence imaging.

\section{Fluorescence Imaging}

Following aptamer incubation, dishes containing the acute slices were placed on the stage of a Zeiss 710 con- focal laser scanning microscope. TD05-488 was imaged with $488 \mathrm{~nm}$ excitation and 505-525 $\mathrm{nm}$ emission. Red fluorescent protein was imaged with $560 \mathrm{~nm}$ excitation and 575-640 $\mathrm{nm}$ emission. Images were obtained with a Zeiss 20x/0.8 NA dry objective and confocal aperture of 1 Airy unit. The frame size was set to sample at the Nyquist rate. The laser and gain were set to fill the dynamic range of the photomultiplier tube in regions of strong fluorescence, and settings were maintained for all regions within each acute slice. For each slice, we imaged 7 regions of interest (ROIs) of $141 \times 141 \mu \mathrm{m}$ from the area of tumor implantation and 3 ROIs from contralateral normal brain.

\section{Histology and IHC}

Rodent xenograft brains containing CNS B-cell lymphoma or glioma were fixed in $4 \%$ paraformaldehyde and then embedded in paraffin. The brains were sectioned $(8$ lymphoma sections, 8 glioma sections) and stained with $\mathrm{H} \& \mathrm{E}$. Additionally, the sections were counterstained for CD20 antibody $(0.93 \mathrm{mg} / \mathrm{L}$, Leica Biosystems Inc.) with a Bond III automated slide stainer (Lecia Biosystems Inc.). Sections were mounted on slides with optical glass 

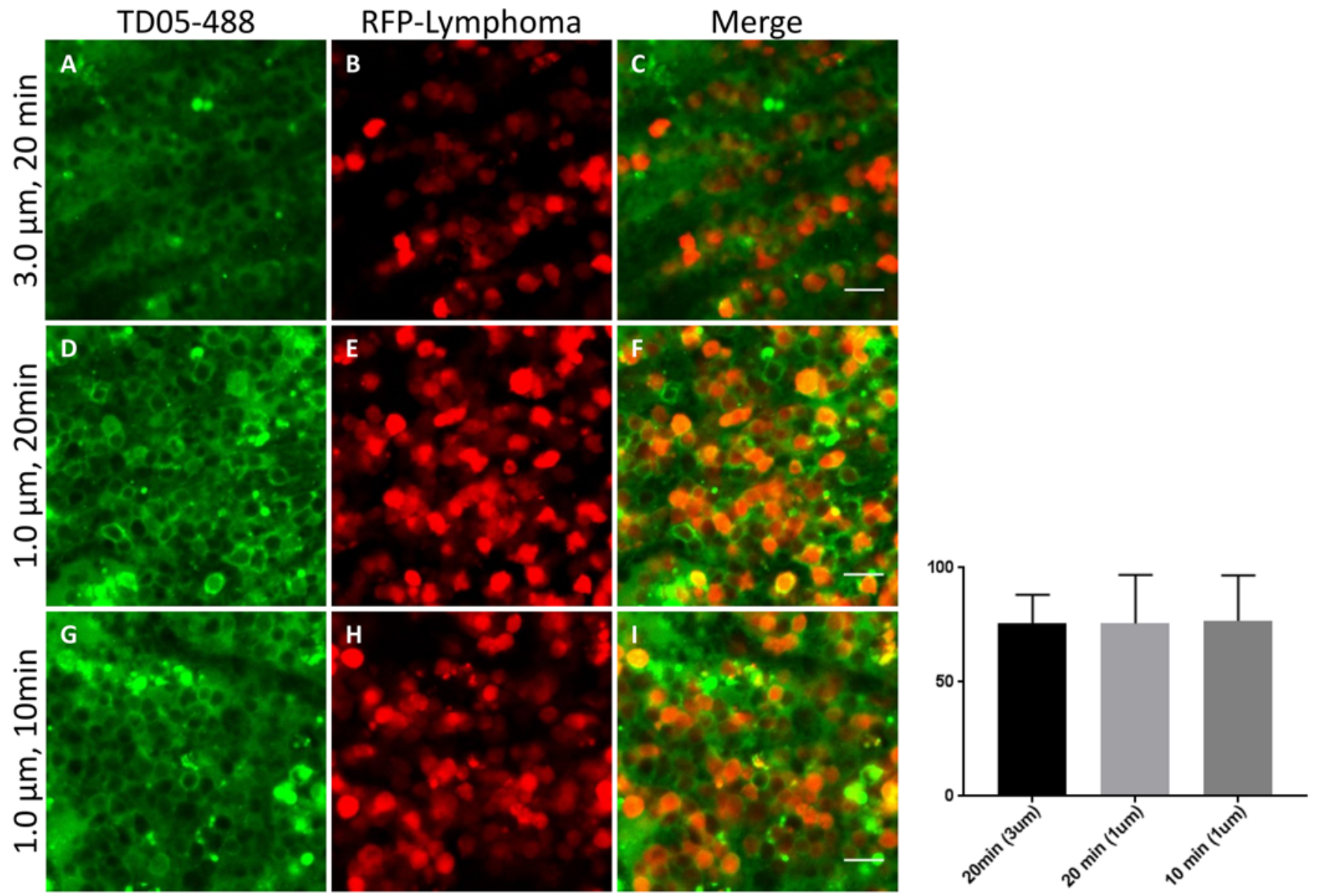

FIG. 2. Twenty-minute staining with 3.0- $\mu \mathrm{M}(\mathbf{A}-\mathbf{C})$ and $1.0-\mu \mathrm{M}(\mathbf{D}-\mathbf{F})$ concentrations did not identify significantly more lymphoma cells than 10-minute staining with 1.0- $\mu \mathrm{M}$ TD05-488 (G-I). Bar $=20 \mu \mathrm{m}$.

and imaged with a 20× objective from an Olympus BX51 brightfield microscope (Olympus America).

\section{Image Analysis}

All image processing was completed utilizing linear functions in ImageJ (US National Institutes of Health). ${ }^{19}$ Regions of interest were randomly selected from areas of green fluorescence within each image. For each ROI, the number of cells expressing RFP and the number of cells with ring-like green fluorescence were quantified utilizing stereology approaches, as previously described., ${ }^{3,20}$ The percent of tumor cells labeled with the fluorescent aptamer was determined by quantifying the percent of RFPexpressing cells labeled with green fluorescence per ROI.

\section{Clinician Image Evaluation}

A file of 27 random ROIs was generated from images obtained from regions of CNS lymphoma, glioma, and normal brain incubated with $1-\mu \mathrm{M}$ TD05-488 for $10 \mathrm{~min}-$ utes. As a reference, an image of CNS lymphoma labeled with a fluorescent antibody was included. This file was distributed to neurosurgeons and pathologists, and their ability to distinguish CNS lymphoma from controls was quantified. The clinicians were blinded to the diagnosis of each image.

\section{Statistical Analysis}

Statistical analysis was performed with Prism version 7 for Windows (GraphPad Software). Data containing three or more groups were analyzed with ANOVA. A post hoc Tukey's multiple comparisons test was utilized to assess for significance in the difference between means. A Student t-test was implemented to identify differences when only two groups were compared. The alpha value was set for 0.05 for all tests. A logit model for data analysis was considered. However, percentages were maintained for simplicity and for a lack of extremes in the data set.

\section{Results}

\section{Optimization of Staining Time and Aptamer Concentration}

To develop an aptamer staining protocol that could identify CNS lymphoma quickly after frozen section, we first evaluated the staining efficacy of three concentrations of fluorescent aptamer at 20 minutes: $0.3,1.0$, and $3.0 \mu \mathrm{M}$. The 3.0- and 1.0- $\mu \mathrm{M}$ concentrations provided strong subjective staining of lymphoma cells and labeled $78 \%$ versus 

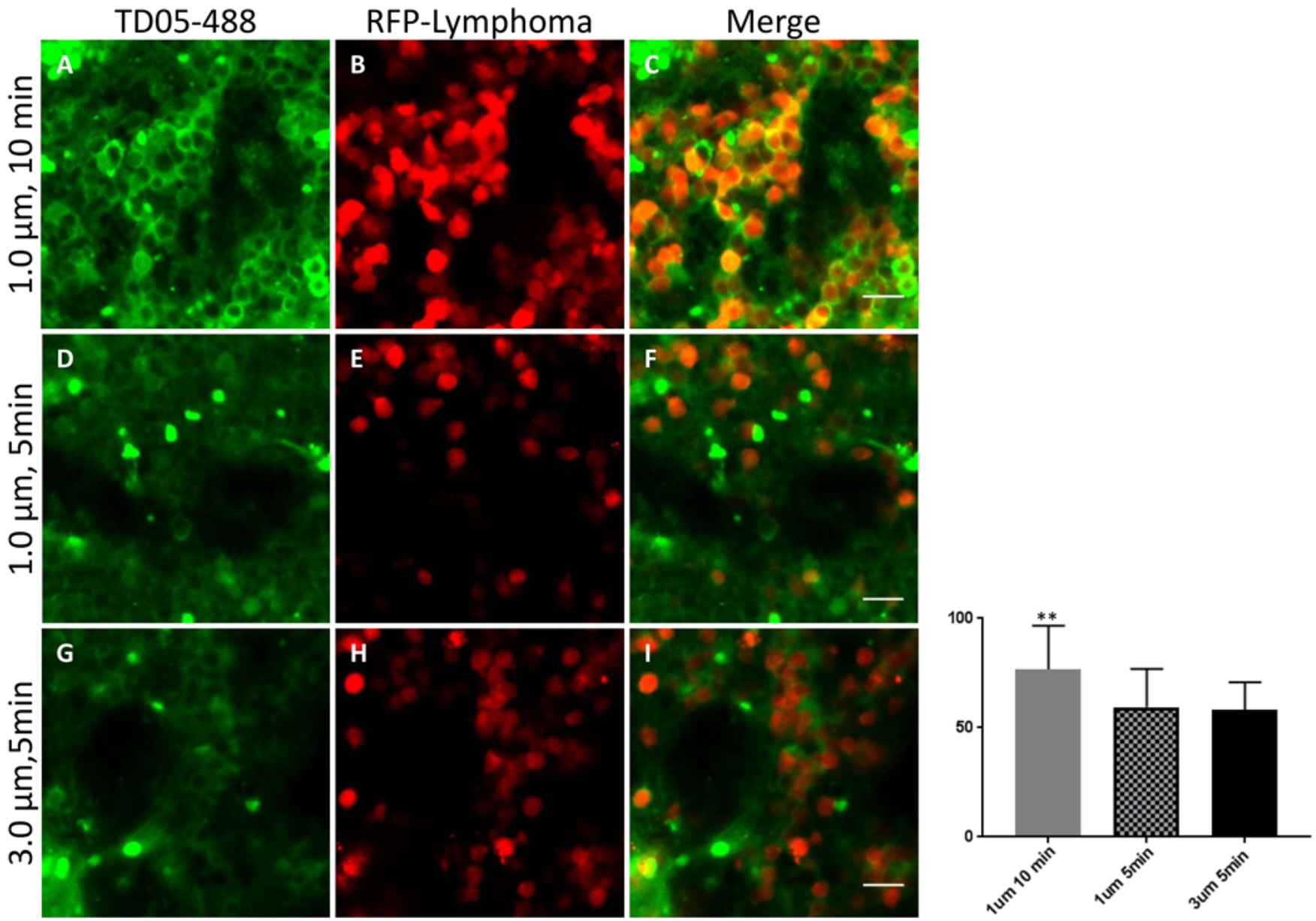

FIG. 3. Ten-minute staining with 1.0- $\mu \mathrm{M}$ TD05-488 (A-C) identified a significantly greater percentage of tumor cells than the 5-minute staining with the $1.0-\mu \mathrm{M}(\mathbf{D}-\mathbf{F})$ and $3.0-\mu \mathrm{M}(\mathbf{G}-\mathbf{I})$ concentrations. Bar $=20 \mu \mathrm{m}$. ${ }^{* *} p<0.001$.

$77 \%$ of cells $(\mathrm{p}=0.99)$, respectively, at this time point. However, $0.3-\mu \mathrm{M}$ TD05-488 labeled only $47 \%$ of tumor cells, which was significantly less than 3.0- and $1.0-\mu \mathrm{M}$ TD05-488 at 20 minutes $(\mathrm{p}<0.001$; Fig. 1). We tested the staining efficacy of $1.0 \mu \mathrm{M}$ at 10 minutes and found no significant difference compared to 1.0 and $3.0 \mu \mathrm{M}$ at 20 minutes ( $\mathrm{p}=0.97$; Fig. 2). Given that $1.0 \mu \mathrm{M}$ provided interpretable staining at 10 minutes, we next evaluated the staining efficacy of 3.0 and $1.0 \mu \mathrm{M}$ at 5 minutes. Compared to $1.0 \mu \mathrm{M}$ at 10 minutes, we noted significantly less labeling with a 5-minute protocol utilizing these concentrations of TD05-488 ( $p<0.001$; Fig. 3). Given these study findings, we selected an aptamer concentration of $1.0 \mu \mathrm{M}$ with a staining time of 10 minutes followed by a 1-minute wash for testing with control samples; this protocol was most efficient, labeling $76.7 \% \pm 15.1 \%$ of lymphoma cells. For clarity, fluorescent artifacts are identified in Fig. 1. As previously reported, these artifacts are small areas of high fluorescent intensity that lack a structural ring-like staining pattern. ${ }^{3}$ Artifacts are present but not labeled in Figs. 2 and 3 .

\section{Astrocytoma}

To evaluate nonspecific staining of our fluorescent ap- tamer on a negative control tumor, we generated acute slices from rodents implanted with human U251 glioma cells expressing RFP. Three acute slices from each of 3 rodents were incubated for 10 minutes with $1.0-\mu \mathrm{M}$ TD05488. Seven ROIs were imaged from each acute slice, and cellular fluorescence was quantified. We found less than $1 \%$ fluorescence staining resembling CD20-positive lymphoma staining in these samples, showing a 10-minute staining protocol with 1.0- $\mu \mathrm{M}$ TD05-488 yielded a falsepositive rate of $0.81 \% \pm 1.75 \%$ and labeled significantly fewer astrocytoma cells than lymphoma cells $(\mathrm{p}<0.001$; Fig. 4).

\section{Standard Histology}

Brain sections from CNS B-cell lymphoma and glioma xenografts were processed for traditional $\mathrm{H} \& \mathrm{E}$ and CD20 antibody staining. The H \& E staining showed regions of hypercellular tumor in lymphoma and glioma specimens (Supplemental Fig. 2A and E). Lymphoma sections showed strong CD20 staining (Supplemental Fig. 2B and C), whereas glioma samples lacked CD20 staining (Supplemental Fig. 2F and G). High magnification revealed a ring-like staining pattern of CD20 in lymphoma samples (Supplemental Fig. 2C) and a similar aptamer 

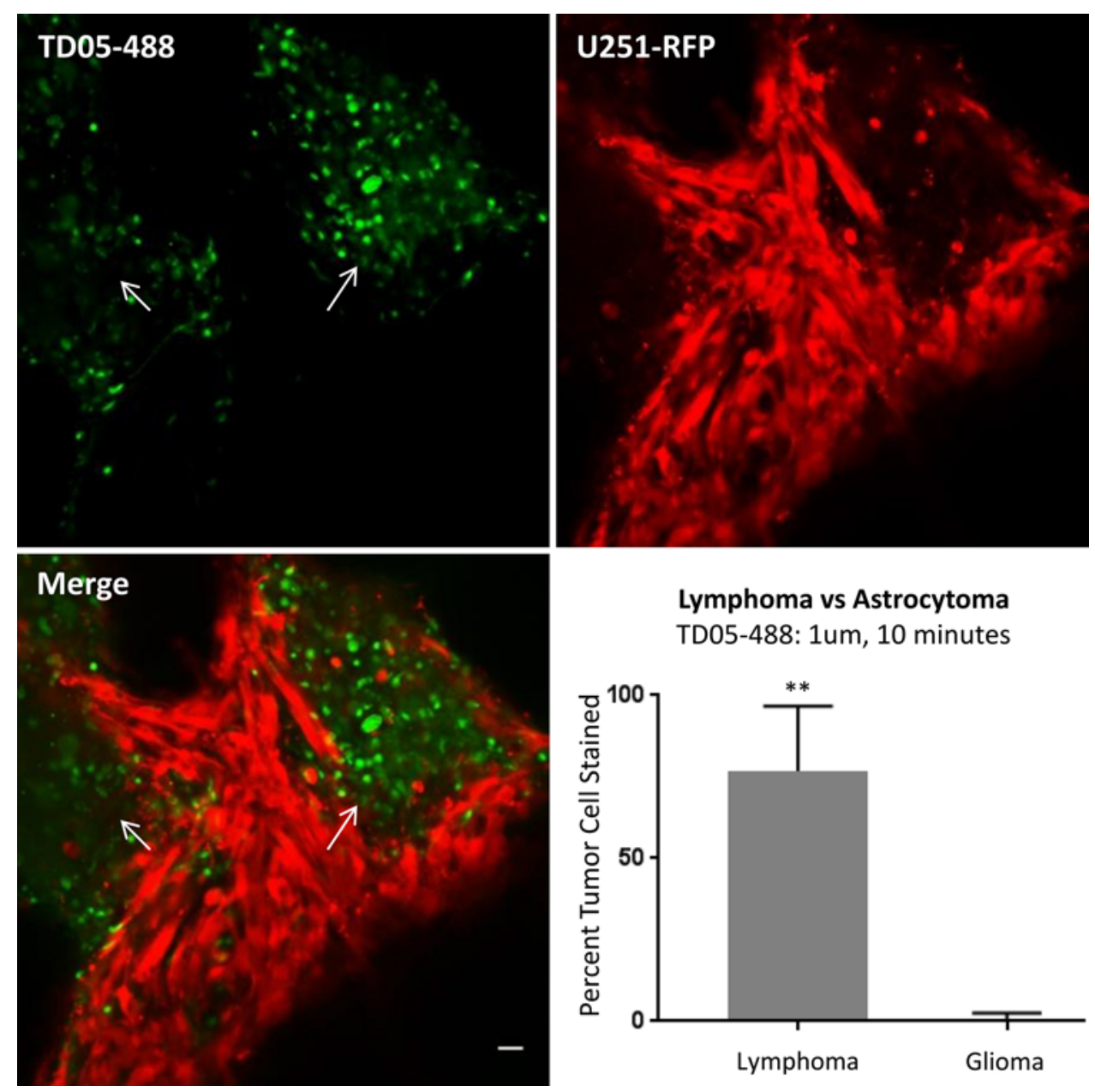

FIG. 4. Astrocytoma negative control. Ten-minute staining protocol with $1-\mu \mathrm{M}$ TD05-488 yielded a false-positive rate of $0.81 \% \pm$ $1.75 \%$ from astrocytoma xenograft biopsies. Note the areas with fluorescent artifacts that lack a ring-like staining pattern (arrows). Bar $=20 \mu \mathrm{m} .{ }^{* *} p<0.001$.

staining pattern of RFP-expressing B-cell lymphoma cells (Supplemental Fig. 2D). Glioma samples lacked the ring-like staining pattern visualized from lymphoma cells (Supplemental Fig. 2G and H).

\section{Additional Controls: Normal Brain and Nonspecific Aptamer}

For additional controls, we evaluated a 10-minute staining protocol with 1.0- $\mu \mathrm{M}$ TD05-488 on normal brain and assessed the staining of a nonspecific Alexa Fluor 488conjugated aptamer on positive control biopsies. Our nonspecific fluorescent aptamer did not generate appreciable staining in lymphoma acute slices incubated in $1.0 \mu \mathrm{M}$ for 10 minutes $(2.71 \% \pm 3.72 \%$ cells labeled; Fig. $5 \mathrm{~A}$ and $\mathrm{B})$. We found that TD05-488 labeled $0.80 \% \pm 1.27 \%$ cells in normal brain (Fig. 5C and D).

\section{Clinician Image Review}

We evaluated two board-certified neurosurgeons (P.N. and S.Y.) and two fellowship-trained clinical pathologists (J.E. and Hany Osman) in their interpretations of aptamerbased fluorescence images by distributing an image file containing 27 random samples incubated with our 10-minute TD05-488 staining protocol (Supplemental Fig. 1). The images contained ROIs from lymphoma, astrocytoma, and normal brain. Clinicians were asked if each image contained CNS lymphoma or not. Overall, each clinician identified all lymphoma cases correctly. In this analysis, there were no false positives and no false negatives, and the intrarater and interrater correlations showed perfect agreement with a correlation of 1.0.

\section{Discussion}

We describe a truncated fluorescence aptamer providing specific diagnosis of xenograft CNS lymphoma within 11 minutes of biopsy (Fig. 2), a time frame that can provide meaningful intraoperative feedback. Our previous report indicated that a conformational-switching fluorescent aptamer could specifically diagnose CNS lymphoma from rodent xenograft biopsies within 45-60 minutes ${ }^{3}$-faster than IHC but too slow for effective intraoperative feedback.

In this study, we initially hypothesized that a 20 -minute aptamer staining protocol that labeled $80 \%$ of lymphoma 


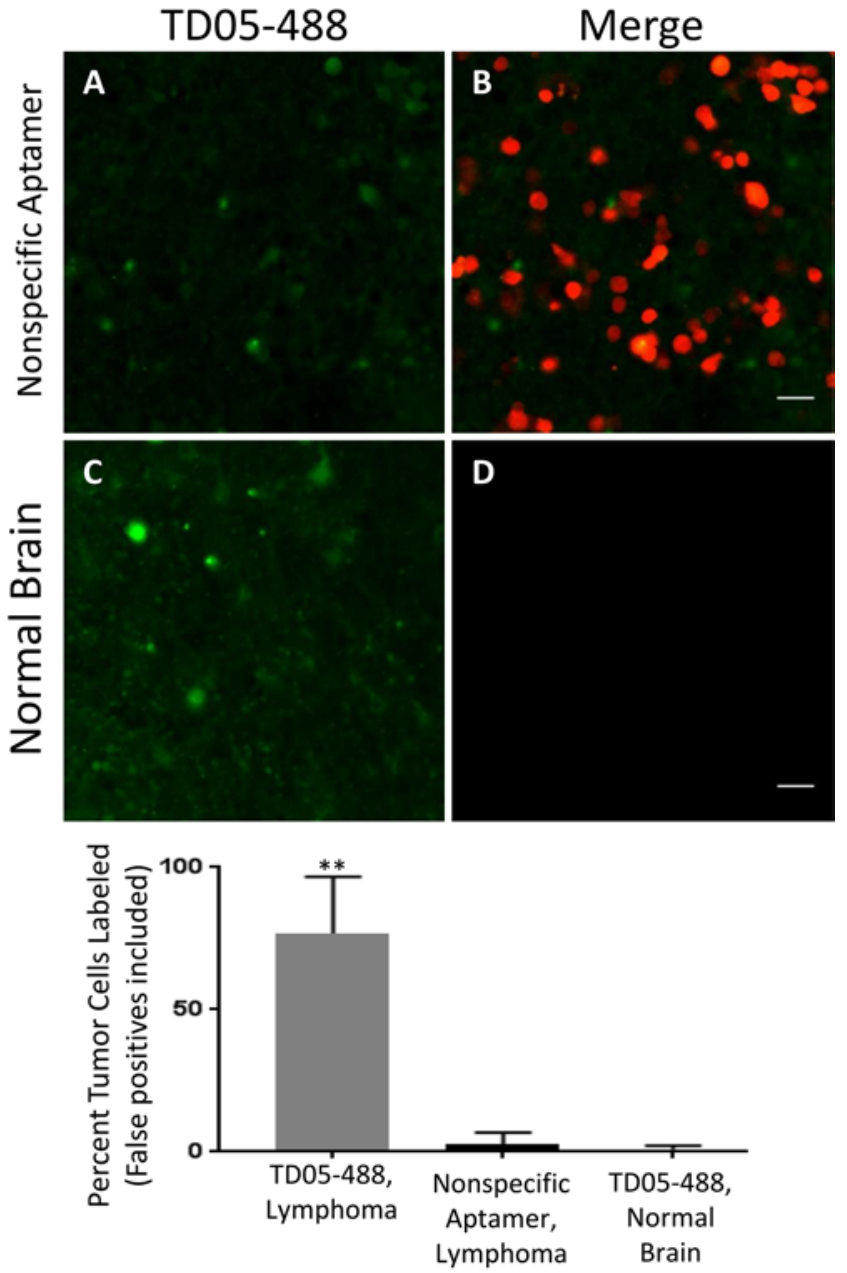

FIG. 5. Nonspecific aptamer and normal brain negative controls. Lymphoma biopsies incubated with a nonspecific fluorescent aptamer (A and B) and normal brain incubated with 1.0- $\mu \mathrm{M}$ TD05-488 (C and D) yielded a false-positive rate of $2.71 \% \pm 3.72 \%$ and $0.80 \% \pm 1.27 \%$, respectively, compared to $76.7 \% \pm 15.1 \%$ of lymphoma cells labeled by TD05-488. Bar $=20 \mu \mathrm{m}$.

cells with a false-positive rate under $10 \%$ would provide histopathological data sufficient to diagnose CNS lymphoma in fresh biopsies. ${ }^{3}$ We evaluated three concentrations of TD05-488 at three times points $(5,10$, and 20 minutes, followed by a 1-minute wash) and found that a $1.0-\mu \mathrm{M}$ staining protocol at 10 minutes labeled $77 \%$ of CNS cells with a false-positive rate under $1 \%$ from negative control astrocytoma biopsies (Fig. 4). Though we did not observe staining of $80 \%$ or greater in positive controls, staining with 1.0- $\mu \mathrm{M}$ TD05-488 at 10 minutes provided consistent differentiation of CNS lymphoma from negative control samples for neurosurgeons and clinical pathologists.

Staining samples with TD05-488 was straightforward compared to the multiple steps required for frozen sections and IHC: tissue samples were placed in solution containing 1.0- $\mu \mathrm{M}$ TD05-488, rinsed for 1 minute, and then immediately imaged. Ex vivo imaging with the Zeiss 710 confocal microscope further improved time to diagnosis by eliminating the sectioning and slide preparation required for frozen sections and IHC. In comparison, an overnight staining protocol was required to complete standard IHC staining for samples viewed with brightfield imaging in this study (Supplemental Fig. 2). Clinical ex vivo confocal imaging is a burgeoning field promising expedited histopathological diagnoses. ${ }^{21,22}$ The development of rapid molecular probes, such as TD05-488, should increase the clinical utility of this imaging modality.

There are barriers to implementing TD05-488 as a clinical diagnostic agent. Though ex vivo confocal microscopy increased the speed of interrogating samples after aptamer incubation, few pathology departments possess this imaging capability, and acquiring these microscopes can be expensive. Currently, fluorescence imaging techniques are showing utility in clinical pathology. As applications for fluorescence diagnostics increase, clinical fluorescence imaging devices capable of optical sectioning, such as confocal microscopes, may become more affordable and commonplace in pathology departments. Additionally, though TD05-488 provided reliable identification of CNS lymphoma from xenograft biopsies, a rigorous clinical trial is required to determine its efficacy on clinical specimens. We identified fluorescent artifacts in a majority of our aptamer-labeled images (Figs. 1 and 4). However, these artifacts did not resemble ring-like CD20 staining and did not influence diagnoses within our blinded image set (Supplemental Fig. 1).

\section{Conclusions}

Intraoperative differentiation of CNS lymphoma from other malignant brain tumors, such as astrocytomas, remains a challenge in neuro-oncology. As a proof of concept, we have shown that ex vivo confocal imaging with the fluorescent aptamer TD05-488 can diagnose CNS lymphoma within 11 minutes of biopsy from xenograft brain tumor models. This procedure was straightforward and required fewer preparation steps than frozen sections or IHC. Clinical application of TD05-488, as well as other similar aptamers, may improve patient care by providing physicians with definitive intraoperative diagnoses.

\section{Acknowledgments}

We thank Margaret Patterson, Michelle Clark, and Kathleen Brown from Abington-Jefferson Memorial Hospital Department of Pathology for assisting with histology for this study. We also thank Hany Osman, MD, a fellowship-trained clinical pathologist, for reviewing images from our 27-ROIs random image file.

This work was supported by the National Institutes of Health grant no. 1R21EB020237-01A1 (P.N. and H.Y.).

\section{References}

1. Ziv E, Durack JC, Solomon SB. The importance of biopsy in the era of molecular medicine. Cancer J. 2016;22(6):418-422.

2. Carnevale J, Rubenstein JL. The challenge of primary central nervous system lymphoma. Hematol Oncol Clin North Am. 2016;30(6):1293-1316

3. Georges JF, Liu X, Eschbacher J, et al. Use of a conformational switching aptamer for rapid and specific ex vivo identification of central nervous system lymphoma in a xenograft model. PLoS One. 2015;10(4):e0123607.

4. Koriyama S, Nitta M, Shioyama T, et al. Intraoperative flow cytometry enables the differentiation of primary central ner- 
vous system lymphoma from glioblastoma. World Neurosurg. 2018;112:e261-e268.

5. Scott BJ, Douglas VC, Tihan T, et al. A systematic approach to the diagnosis of suspected central nervous system lymphoma. JAMA Neurol. 2013;70(3):311-319.

6. Owen CM, Linskey ME. Frame-based stereotaxy in a frameless era: current capabilities, relative role, and the positiveand negative predictive values of blood through the needle. $J$ Neurooncol. 2009;93(1):139-149.

7. Davis ME. Glioblastoma: overview of disease and treatment. Clin J Oncol Nurs. 2016;20(5)(suppl):S2-S8.

8. Fernandes C, Costa A, Osorio L, et al. Current standards of care in glioblastoma therapy. In: De Vleeschouwer S, ed. Glioblastoma. Codon; 2017.

9. Chand P, Amit S, Gupta R, Agarwal A. Errors, limitations, and pitfalls in the diagnosis of central and peripheral nervous system lesions in intraoperative cytology and frozen sections. J Cytol. 2016;33(2):93-97.

10. Tofte K, Berger C, Torp SH, Solheim O. The diagnostic properties of frozen sections in suspected intracranial tumors: a study of 578 consecutive cases. Surg Neurol Int. 2014;5:170.

11. Amraei R, Moradi A, Zham H, et al. A comparison between the diagnostic accuracy of frozen section and permanent section analyses in central nervous system. Asian Pac J Cancer Prev. 2017;18(3):659-666.

12. Raoux D, Duband S, Forest F, et al. Primary central nervous system lymphoma: immunohistochemical profile and prognostic significance. Neuropathology. 2010;30(3):232-240.

13. Ellington AD, Szostak JW. In vitro selection of RNA molecules that bind specific ligands. Nature. 1990;346(6287):818822 .

14. Keefe AD, Pai S, Ellington A. Aptamers as therapeutics. Nat Rev Drug Discov. 2010;9(7):537-550.

15. Hicke BJ, Stephens AW, Gould T, et al. Tumor targeting by an aptamer. J Nucl Med. 2006;47(4):668-678.

16. Mallikaratchy P, Tang Z, Kwame S, et al. Aptamer directly evolved from live cells recognizes membrane bound immunoglobin heavy mu chain in Burkitt's lymphoma cells. Mol Cell Proteomics. 2007;6(12):2230-2238.

17. Mallikaratchy PR, Ruggiero A, Gardner JR, et al. A multivalent DNA aptamer specific for the B-cell receptor on human lymphoma and leukemia. Nucleic Acids Res. 2011;39(6): $2458-2469$.

18. Tang Z, Mallikaratchy P, Yang R, et al. Aptamer switch probe based on intramolecular displacement. J Am Chem Soc. 2008:130(34):11268-11269.

19. Schneider CA, Rasband WS, Eliceiri KW. NIH Image to Image J: 25 years of image analysis. Nat Methods. 2012;9(7): 671-675.

20. Georges JF, Martirosyan NL, Eschbacher J, et al. Sulforhodamine 101 selectively labels human astrocytoma cells in an animal model of glioblastoma. J Clin Neurosci. 2014;21(5): $846-851$.
21. Martirosyan NL, Georges J, Eschbacher JM, et al. Confocal scanning microscopy provides rapid, detailed intraoperative histological assessment of brain neoplasms: experience with 106 cases. Clin Neurol Neurosurg. 2018;169:21-28.

22. Mooney MA, Georges J, Yazdanabadi MI, et al. Immediate ex-vivo diagnosis of pituitary adenomas using confocal reflectance microscopy: a proof-of-principle study. J Neurosurg. 2018;128(4):1072-1075.

\section{Disclosures}

The authors report no conflict of interest concerning the materials or methods used in this study or the findings specified in this paper.

\section{Author Contributions}

Conception and design: Nakaji, Georges, Liu, Feuerstein, Reiser, Anderson, Yan. Acquisition of data: Nakaji, Georges, Qi, Liu, Zhou, Woolf, Anderson, Yocom, Eschbacher, Yan. Analysis and interpretation of data: Nakaji, Georges, Qi, Liu, Woolf, Valeri, Al-Atrache, Belykh, Feuerstein, Preul, Scheck, Reiser, Anderson, Appelt, Yocom, Eschbacher, Yan. Drafting the article: Nakaji, Georges, Qi, Liu, Woolf, Valeri, Al-Atrache, Belykh, Feuerstein, Preul, Scheck, Reiser, Anderson, Yan. Critically revising the article: Nakaji, Georges, Qi, Liu, Zhou, Woolf, Valeri, Al-Atrache, Belykh, Feuerstein, Preul, Scheck, Reiser, Anderson, Appelt, Yocom, Eschbacher, Yan. Reviewed submitted version of manuscript: all authors. Approved the final version of the manuscript on behalf of all authors: Nakaji. Statistical analysis: Nakaji, Georges, Reiser, Yan. Administrative/technical/material support: Nakaji, Feuerstein, Preul, Scheck, Reiser, Anderson, Appelt, Yocom, Eschbacher, Yan. Study supervision: Nakaji, Georges, Feuerstein, Scheck, Anderson, Yan.

\section{Supplemental Information}

\section{Online-Only Content}

Supplemental material is available with the online version of the article.

Supplemental Figs. 1 and 2. https://thejns.org/doi/suppl/ 10.3171/2020.4.JNS192476.

\section{Correspondence}

Peter Nakaji: University of Arizona, College of Medicine, Phoenix, AZ.nakaji@email.arizona.edu. 